\title{
脂肪胺不对称转化新突破
}

徐明华

南方科技大学化学系/深圳格拉布斯研究院, 深圳 518055

E-mail: xumh@sustech.edu.cn

\section{Asymmetric transformation of aliphatic amines: A breakthrough}

\author{
Ming-Hua Xu \\ Department of Chemistry and Shenzhen Grubbs Institute, Southern University of Science and Technology, Shenzhen 518055, China \\ E-mail: xumh@sustech.edu.cn
}

doi: 10.1360/TB-2019-0788

手性脂肪胺是一类极其重要的化合物, 很多天然产 物、手性药物和农药分子中都含有脂肪胺结构, 20 种天然 氨基酸也都是 $\alpha$-羧基取代的脂肪胺, 可以说手性脂肪胺在 生命活动中占据重要地位. 在手性合成中, 直接利用脂肪 胺在过渡金属催化下合成光学活性胺是一个非常挑战的 课题, 主要难点是脂肪胺氮上富电子, 具有较强的Lewis 碱性, 容易与Lewis酸性的过渡金属配位, 从而导致金属 催化剂失活. 过渡金属催化下脂肪胺的不对称转化已经成 为困扰科学家的一个难题.

南开大学周其林和朱守非团队一直致力于开发高效 构筑 $\mathrm{C}-\mathrm{N}$ 键的不对称催化策略, 在过渡金属催化卡宾对 $\mathrm{N}-\mathrm{H}$ 键的不对称插人反应方面 ${ }^{[1]}$ 进行了系统的研究 ${ }^{[2]}$. 2007年, 该研究团队 ${ }^{[3]}$ 使用手性螺环噁唑啉配体铜配合物 催化剂首次实现了金属卡宾对芳胺 $\mathrm{N}-\mathrm{H}$ 键的高对映选择性 插人反应. 2011年, 他们 ${ }^{[4]}$ 又利用手性磷酸和铑配合物双 催化体系成功实现了金属卡宾对酰胺 $\mathrm{N}-\mathrm{H}$ 键的不对称插人 反应. 最近, 该团队 ${ }^{[5]}$ 进一步在极具挑战的金属卡宾对脂 肪胺的 $\mathrm{N}-\mathrm{H}$ 键的不对称插人反应研究中取得突破, 发展了 一种新颖的双催化剂体系, 将极易引起金属催化剂失活的 脂肪胺成功用于 $\alpha$-氨基酸类重要分子的不对称合成中, 为 脂肪胺的不对称转化提供了一个全新的策略, 破解了这个 长期以来困扰不对称催化领域的难题, 研究成果发表在 Science上.

南开大学研究团队发现带一个负电荷的三齿蝎型配 体和一价铜的络合物 $(\mathrm{Tp} * \mathrm{Cu})$ 在过量脂肪胺存在的条件下, 仍然能保持催化活性, 与烷基重氮酸酯作用生成金属卡宾 物种. 进一步以手性氨基硫艮为催化剂, 可以高效实现金 属卡宾对脂肪胺 $\mathrm{N}-\mathrm{H}$ 键的高对映选择性插人, 得到一系列
高光学纯度的手性 $\alpha$-氨基酸衍生物(图 1(a)). 该方法具有很 好的底物普适性, 同时适用于一级胺和二级胺(图1(b)), 反 应大多能取得优秀的收率(高达99\%)和对映选择性(高达 97\% ee). 尤其值得一提的是, 利用该方法可以方便地实现 多种药物及生物活性分子中 (如阿莫沙平、曲美他溙、沃替 西汀等)相应手性 $\alpha$-氨基酸结构单元的高效立体选择性合 成(图1(c)).

应用在线红外光谱技术测定了反应动力学, 发现铜配 合物 $(\mathrm{Tp} * \mathrm{Cu})$ 和重氮底物为一级反应, 手性硫脉催化剂 (CAT) 为负一级反应, 脂肪胺为零级反应, 由此推断反应 的决速步骤是重氮分解生成卡宾. 接着他们利用核磁共振 和紫外光谱技术, 证明了金属铜配合物和脂肪胺几乎不存 在相互作用, 而手性硫䐂催化剂的硫原子与铜有较强的配 位作用. 他们认为这是由于配体带有一个负电荷，与正一 价铜结合后生成中性的铜配合物, 使铜变得更“软”。根据 软硬酸碱理论, 它很难与较“硬”的脂肪胺作用. “以柔克 刚”, 这是该反应能发生的关键.

在立体化学决定步骤的研究中, 他们创造性地提出: 手性氨基硫艮催化剂既可以用氨基部分接受质子, 又可以用 硫腿部分给出质子，这样“一推一拉”实现了质子转移过程的 手性控制. 他们还发现手性胺基硫腿催化剂对铜的配位虽然 在一定程度减缓了反应速度, 但同时也增加了硫㽷催化剂的 $\operatorname{Br} \varnothing n s t e d$ 酸性 $\left(\mathrm{p} K_{\mathrm{a}}\right.$ 从 13.60降低到了9.84), 提高了质子转移的 效率(图2(a)). 结合密度泛函理论(DFT)计算, 给出了质子转 移的手性控制模型(图2(b)), 并指出氢键作用和非共价的弱 相互作用是反应能取得高对映选择性的主要原因.

南开大学周其林和朱守非团队 ${ }^{[5]}$ 发展了一种新颖的 双催化剂体系, 利用非手性铜催化剂和手性有机催化剂的 
(a)

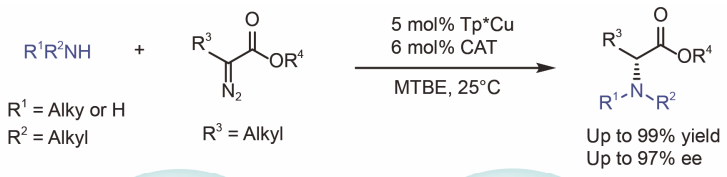

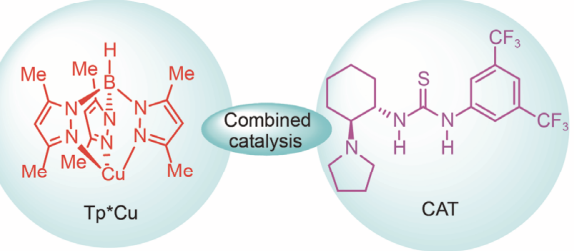

(b)

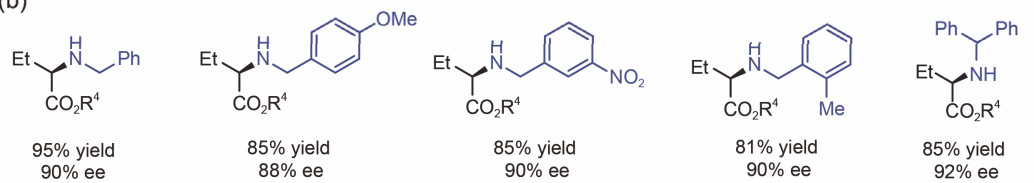

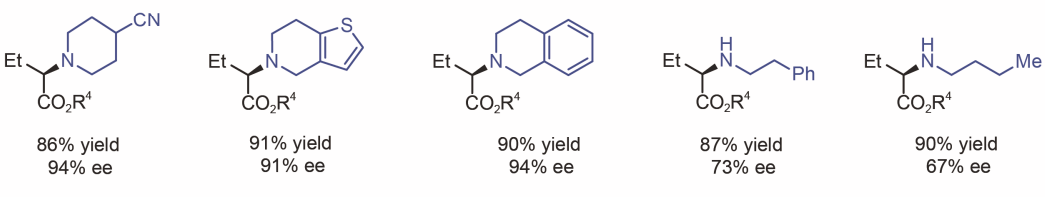

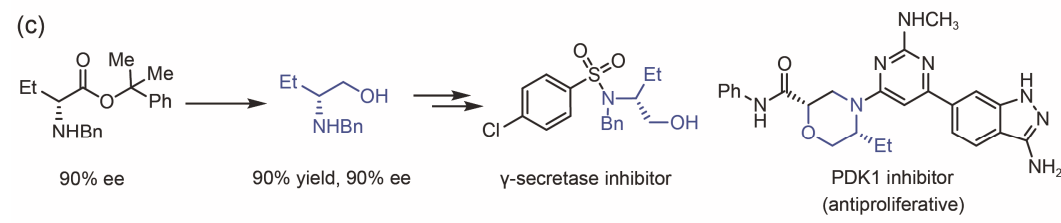<smiles>CCC(C(=O)Nc1cc(C(=O)Nc2ccc(-c3ccccc3)cc2)ccc1OC)N1CCOCC1</smiles>

图 1 (网络版彩色)双催化剂策略用于卡宾对脂肪胺 $\mathrm{N}-\mathrm{H}$ 键的对映选择性插人

Figure 1 (Color online) Enantioselective carbene insertion to N-H bonds of aliphatic amines by using two catalysts strategy

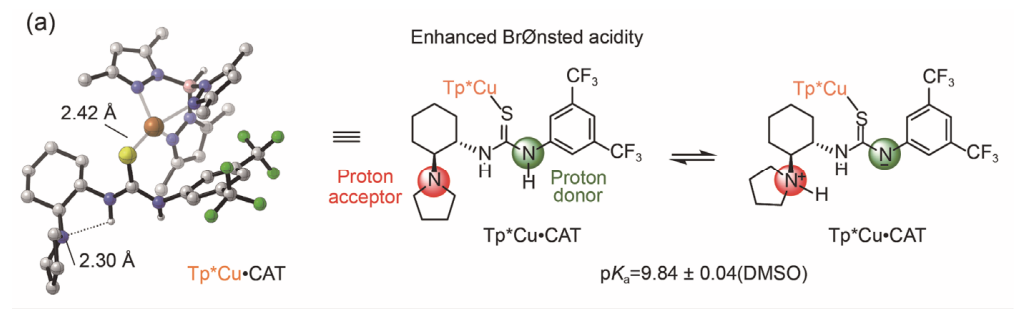

(b)
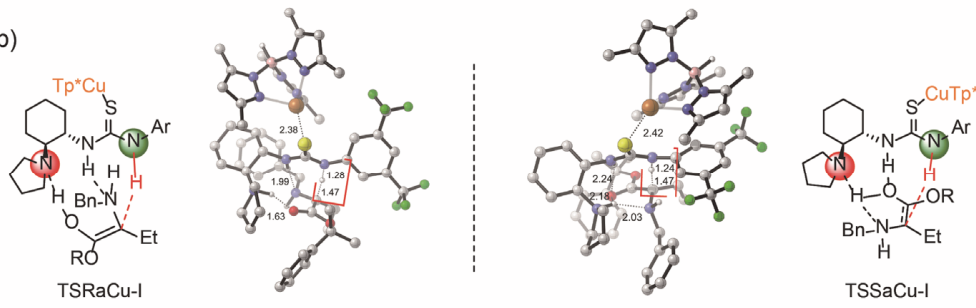

$\Delta G^{\ddagger}=2.8 \mathrm{kcal} / \mathrm{mol}$

$\Delta G^{\star}=7.7 \mathrm{kcal} / \mathrm{mol}$

图 2 (网络版彩色) $\mathrm{Tp} * \mathrm{Cu}$ 对氨基硫脲的活化模型及质子转移的手性控制模型

Figure 2 (Color online) Computational modeling of the $\mathrm{Tp} * \mathrm{Cu} \cdot \mathrm{CAT}$ complex and the transition state for enantio-determining proton-transfer 
协同催化, 成功实现了铜卡宾对脂肪胺 $\mathrm{N}-\mathrm{H}$ 键的高对映选 择性插人. 该研究不仅解决了一个长期以来困扰科学家的 脂肪胺不能用于金属卡宾不对称插人反应的难题, 而且为 强配位性底物参与的过渡金属催化不对称反应提供了一
个潜在的通用策略. 该研究发展的方法可以高效、高对映 选择性地合成结构多样的天然氨基酸或非天然氨基酸衍 生物, 有望在肽类药物以及相应的含脂肪胺结构的手性药 物合成中得到广泛应用.

\section{参考文献}

1 Ford A, Miel H, Ring A, et al. Modern organic synthesis with $\alpha$-diazocarbonyl compounds. Chem Rev, 2015, 115: 9981-10080

2 Zhu S F, Zhou Q. Transition-metal-catalyzed enantioselective heteroatom-hydrogen bond insertion reactions. Acc Chem Res, 2012, 45: $1365-1377$

3 Liu B, Zhu S F, Zhang W, et al. Highly enantioselective insertion of carbenoids into N-H bonds catalyzed by copper complexes of chiral spiro bisoxazolines. J Am Chem Soc, 2007, 129: 5834-5835

4 Liu B, Zhu S F, Xie X L, et al. Asymmetric N-H insertion reaction cooperatively catalyzed by rhodium and chiral spiro phosphoric acids. Angew Chem Int Ed, 2011, 50: 11483-11486

5 Li M L, Yu J H, Li Y T, et al. Highly enantioselective carbene insertion into N-H bonds of aliphatic amines. Science, 2019, 366: 990-994 